\title{
MIXED CONVECTIVE FULLY DEVELOPED FLOW IN A VERTICAL CHANNEL IN THE PRESENCE OF THERMAL RADIATION AND VISCOUS DISSIPATION
}

\author{
K.V. PRASAD* \\ Department of Mathematics, VSK University \\ Vinayaka Nagar, Ballari-583 104, Karnataka, INDIA \\ E-mail: prasadkv2007@gmail.com \\ P. MALLIKARJUN \\ Department of Studies and Research in Mathematics \\ Tumkur University \\ Tumkur, 572 103, INDIA \\ H. VAIDYA \\ Department of Mathematics \\ SSA Government First Grade College \\ Ballari -583 101, Karnataka, INDIA
}

\begin{abstract}
The effect of thermal radiation and viscous dissipation on a combined free and forced convective flow in a vertical channel is investigated for a fully developed flow regime. Boussinesq and Roseseland approximations are considered in the modeling of the conduction radiation heat transfer with thermal boundary conditions (isothermal-thermal, isoflux-thermal, and isothermal-flux). The coupled nonlinear governing equations are also solved analytically using the Differential Transform Method (DTM) and regular perturbation method (PM). The results are analyzed graphically for various governing parameters such as the mixed convection parameter, radiation parameter, Brinkman number and perturbation parameter for equal and different wall temperatures. It is found that the viscous dissipation enhances the flow reversal in the case of a downward flow while it counters the flow in the case of an upward flow. A comparison of the Differential Transform Method (DTM) and regular perturbation method (PM) methods shows the versatility of the Differential Transform Method (DTM). The skin friction and the wall temperature gradient are presented for different values of the physical parameters and the salient features are analyzed.
\end{abstract}

Key words: mixed convection, thermal radiation, viscous dissipation, Differential Transform Method (DTM), Perturbation Method (PM).

\section{Introduction}

The effects of thermal radiations have a very significant application in space technology. There are a few important industrial applications of radiation such as effect on free flows, water evaporation from open reservoirs, heating and cooling of chambers, solar power technology solar fans, solar collectors, photo chemical reactors. Moreover, mixed convection in a vertical parallel-plate channel is of considerable importance in technological industries, such as in heated rooms or reactor configurations, where temperature differences give rise to complicated flow patterns. In fact, it is known that heat exchangers technology often involves convective flows in vertical channels, where these flows in most cases imply thermal conditions of

\footnotetext{
* To whom correspondence should be addressed
} 
uniform heating of channel walls either by isothermal or isoheat flux boundary conditions. Interest in such a study is, therefore, due to its applications in heat exchangers technology, for example, in the design of cooling and solar energy collection systems, etc. In view of these applications, a theoretical study of fully developed, mixed convection in a vertical channel was conducted by Aung and Worku [1] analyzed temperature and velocity profiles for the vertical parallel flow of fully developed regime, whereas Zanchini [2] and Barletta [3] considered thermal boundary conditions and investigated the effects of viscous dissipation on mixed convection flow in a vertical channels. An analytical solution for fully-developed, steady-state, laminar mixed convection with heat and mass transfer between vertical parallel plates was developed by Boulama and Galunis [4]. Further, Barletta et al. [5] discussed a dual mixed convection flow problem in a vertical parallelplate channel. Recently, Prasad et al. [6] studied the effects of temperature-dependent transport properties on the fully developed free and forced MHD convection flow in a vertical channel.

All the above analyses on mixed convection flow in vertical channels are based on the assumption that the effect of thermal radiation within the fluid is negligible. Considering the applications of thermal radiation several researchers studied the combined radiative and convective flow and heat transfer aspects (Refer for details to: Raptis [7], Bakier [8], Raptis and Perdikis [9], Grosan and Pop [10], Bég et al. [11], Ghosh and Bég [12].) and analyzed the thermal radiation effect on a fully developed mixed convection flow in a vertical channel. The excellent review of Özişik [13] on heat transfer by simultaneous radiation and convection provides assistance to many researchers to continue their work in this field.

The objective of this paper is to solve the problem of a fully developed combined mixed convection and radiation effect in a vertical channel, with the effect of viscous dissipation and different thermal boundary conditions using a semi-numerical-analytical method called Differential Transformation Method (DTM) (See Zhou [15], Bert [16], Chu and Chen [17], Chu and Lo [18], Joneidi et al. [19], Rashidi et al. [20], Yaghoobi and Torabi[21] ). This method obtains a solution in the form of a polynomial. In fact this can be seen in the next section where the concept of differential transform is briefly described. The computed results for the flow and heat transfer characteristics are analyzed and show that the fluid flow is appreciably influenced by the sundry parameters. It is expected that the results obtained will not only provide useful information for industrial application, but also complement previous studies.

\section{Governing equations}

A Cartesian coordinate system is chosen such that the $x$-axis is parallel to the gravitational acceleration vector $\boldsymbol{g}$, but in the opposite direction. The $y$-axis is orthogonal to the channel walls, and the origin of the axes is such that the positions of the channel walls are at $Y=-L / 2$ and $Y=L / 2$, respectively. (See Fig.1 for details).

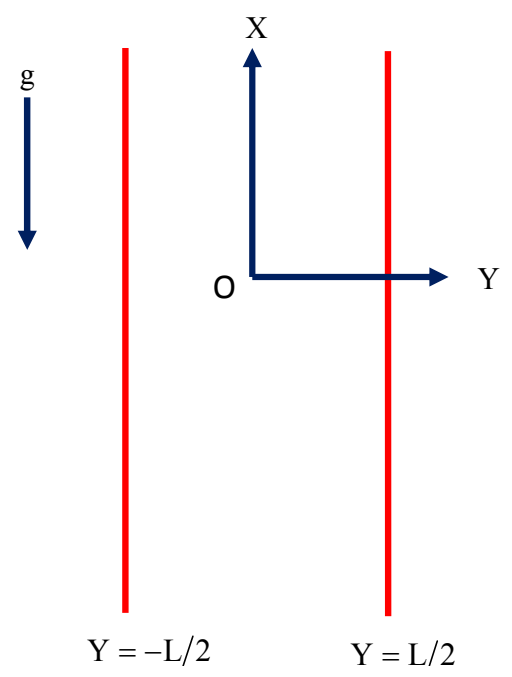

Fig.1. Physical configuration. 
The fluid velocity vector $\boldsymbol{v}(u, v)$ is assumed to be parallel to the $X$-axis, so that the only $U$ does not vanish. The Boussinesq and Roseseland approximation are employed. The fluid rises in the duct driven by buoyancy forces. Hence, the flow is due to the difference in temperature and in the pressure gradient. The fluid properties are the thermal conductivity; the dynamic viscosity and the thermal expansion coefficient are considered as constant. The flow being fully developed the following relations can be applied here

$$
V=0, \quad \frac{\partial V}{\partial Y}=0, \quad \frac{\partial P}{\partial Y}=0
$$

where $P$ is the fluid pressure. Therefore, the continuity Eq. gives $\partial U / \partial X=0$. One can thus conclude that $U$ does not depend on $X$, i.e., $U=U(Y)$. Under these assumptions the momentum and energy equations for the flow and heat transfer can be written as (See Grosan and Pop for details [10])

$$
\begin{aligned}
& g \beta\left(T-T_{0}\right)-\frac{1}{\rho_{0}} \frac{d P}{d X}+v \frac{d^{2} U}{d Y^{2}}=0 . \\
& \alpha \frac{d^{2} T}{d Y^{2}}+\frac{v}{C_{p}}\left(\frac{d U}{d Y}\right)^{2}-\frac{1}{\rho_{0} C_{p}} \frac{d q_{R}}{d Y}=0
\end{aligned}
$$

where $T$ is the fluid temperature, $\beta$ is the thermal expansion coefficient, $v$ is the kinematic viscosity, $\rho_{0}$ is the characteristic density of the fluid, $\alpha$ is the thermal diffusivity, $C_{p}$ is the specific heat at constant pressure and ${ }_{,} q_{R}$ is the radiation heat flux and $T_{0}$ is the inlet temperature. We assume that $q_{R}$ under the Rosseland approximation is in the following form.

$$
q_{R}=-\left(\frac{4 \sigma}{3 \chi}\right) \frac{\partial T^{4}}{\partial Y}
$$

where $\sigma$ is he Stefan-Boltzman constant and $\chi$ is the mean absorption coefficient. The wall at $Y=-L / 2$ has the given uniform temperature $T_{1}$, while the wall at $Y=L / 2$ is subjected to a uniform temperature $T_{2}$, where $T_{2} \geq T_{1}$. These boundary conditions are compatible with Eq.(2.2) if and only if $d P / d X$ is independent of $X$. Therefore, there exists a constant $A$ such that

$$
\frac{d P}{d X}=A
$$

Equations (2.2) and (2.3) give a differential equation for $U$, as

$$
\frac{d^{4} U}{d Y^{4}}-\frac{g \beta}{\alpha C_{p}}\left(\frac{d U}{d Y}\right)^{2}+\frac{g \beta}{\alpha v \rho_{0} C_{p}} \frac{d q_{R}}{d Y}=0 .
$$

The boundary conditions of $U$ are both the no slip conditions

$$
U\left(-\frac{L}{2}\right)=U\left(\frac{L}{2}\right)=0
$$


and the thermal boundary conditions which are given by using Eqs (2.2) and (2.5)

$$
\begin{aligned}
& \left.\frac{d^{2} U}{d Y^{2}}\right|_{Y=-L / 2}=\frac{A}{\mu}-\frac{\beta g\left(T_{1}-T_{0}\right)}{v}, \\
& \left.\frac{d^{2} U}{d Y^{2}}\right|_{Y=L / 2}=\frac{A}{\mu}-\frac{\beta g\left(T_{2}-T_{0}\right)}{v} .
\end{aligned}
$$

The non-dimensional parameters are

$$
\begin{aligned}
& u=\frac{U}{U_{0}} ; \quad \theta=\frac{T-T_{0}}{\Delta T} ; \quad y=\frac{Y}{D} ; \quad \mathrm{Gr}=\frac{g \beta \Delta T D^{3}}{v^{2}} ; \quad \lambda=\frac{\mathrm{Gr}}{\mathrm{Re}} ; \\
& R_{T}=\frac{T_{2}-T_{1}}{\Delta T} ; \quad \operatorname{Re}=\frac{U_{0} D}{v} ; \quad \mathrm{Br}=\frac{\mu U_{0}^{2}}{k \Delta T}, \quad E^{2}=\frac{C D^{2}}{k}
\end{aligned}
$$

where $D=2 L$ is the hydraulic diameter. The reference velocity $U_{0}$ and the reference temperature $T_{0}$ are given by

$$
U_{0}=-\frac{A D^{2}}{48 \mu} ; \quad T_{0}=\frac{T_{1}+T_{2}}{2} . \text { (See Baytas et al. [22]). }
$$

The reference temperature difference $\Delta T$ is given by

$$
\begin{array}{ll}
\Delta T=T_{2}-T_{1} \quad \text { if } \quad T_{1}<T_{2}, \\
\Delta T=\frac{v^{2}}{C_{p} D^{2}} \quad \text { if } & T_{1}=T_{2} .
\end{array}
$$

The dimensionless parameter $R_{T}$ is zero for symmetric heating $\left(T_{1}=T_{2}\right)$ and is one for asymmetric heating $\left(T_{1}<T_{2}\right)$. By using non-dimensional parameters, Eqs (2.3), (2.6) and (2.7a) to (2.7c) become

$$
\begin{aligned}
& \frac{d^{2} \theta}{d y^{2}}+\operatorname{Br}\left(\frac{d u}{d y}\right)^{2}-E^{2} \theta=0 \\
& \frac{d^{4} u}{d y^{4}}-E^{2} \frac{d^{2} u}{d y^{2}}-\lambda \operatorname{Br}\left(\frac{d u}{d y}\right)^{2}-48 E^{2}=0 \\
& u\left(-\frac{1}{4}\right)=u\left(\frac{1}{4}\right)=0,\left.\quad \frac{d^{2} u}{d y^{2}}\right|_{y=-\frac{1}{4}}=-48+\frac{R_{T} \lambda}{2},\left.\quad \frac{d^{2} u}{d y^{2}}\right|_{y=\frac{1}{4}}=-48-\frac{R_{T} \lambda}{2} .
\end{aligned}
$$


Using Eqs (2.8) and (2.9) in Eq.(2.2) we obtain the temperature field as

$$
\theta=-\frac{1}{\lambda}\left(48+\frac{d^{2} u}{d y^{2}}\right)
$$

\section{Solutions}

\section{Special cases}

If the viscous dissipation is negligible such that $\mathrm{Br}=0$, the dimensionless temperature $\theta$ and dimensionless velocity $u$ are uncoupled. In this case, the solution of Eq.(2.13) using the boundary conditions Eq.(2.14), the velocity field becomes

$$
u=\frac{3}{2}-24 y^{2}+\frac{2 R_{T} \lambda}{E^{2}}\left(y-\frac{\sinh (E y)}{4 \sinh (E / 4)}\right) .
$$

Substituting the above velocity field in Eq.(2.15) we get the temperature equation as

$$
\theta=\frac{R_{T}}{2} \frac{\sinh (E y)}{\sinh (E / 4)} .
$$

If the radiation parameter is not considered, i.e., $(E=0)$, the velocity field becomes

$$
u=\left(\frac{R_{T} \lambda y}{3}+24\right)\left(\frac{1}{16}-y^{2}\right)
$$

and the temperature field becomes

$$
\theta=2 R_{T} y
$$

Which corresponds to the velocity and temperature fields determined by Aung and Worku [23]. In case of asymmetric heating, when buoyancy forces are dominated $(\lambda \rightarrow \pm \infty)$ Eqs (2.16) and (2.18a) give

$$
\begin{aligned}
& \frac{u}{\lambda}=\frac{1}{E^{2}}\left(2 y-\frac{\sinh (E y)}{2 \sinh (E / 4)}\right), \\
& \frac{u}{\lambda}=\frac{y}{3}\left(\frac{1}{16}-y^{2}\right),
\end{aligned}
$$

which is Batchelor's [24] velocity field for free convection. When buoyancy forces are negligible and viscous dissipation is relevant, i.e., $\lambda=0$, so that a purely forced convection occurs, solutions of Eqs (2.13) and (2.12) become

$$
u=\frac{3}{2}-24 y^{2}
$$




$$
\theta=-\frac{144 B r}{E^{2} \cosh (E / 4)}\left(\frac{32}{E^{2}}+1\right) \cosh (E y)+\frac{R_{T}}{\sinh (E / 4)} \sinh (E y)+\frac{2304 B r}{E^{2}} y^{2}+\frac{4608 B r}{E^{4}} .
$$

Solutions of Eqs (2.13) and (2.12) for a clear viscous fluid in the absence of buoyancy force and radiation effect lead to the Hagen-Poiseuille velocity profile

$$
u=24\left(\frac{1}{16}-y^{2}\right)
$$

and

$$
\theta=-192 \mathrm{Br} y^{4}+2 R_{T} y+\frac{3 \mathrm{Br}}{4}
$$

which agrees with the results obtained by Cheng and $\mathrm{Wu}$ [25] in the case of forced convection with asymmetric heating.

\subsection{Differential transform method}

The differential transformation method, which is based on Taylor's series expansion, provides a simple approach to derive high order Taylor's coefficient by iterative procedures. Using the differential transformation technique, the ordinary differential equations can be transformed into algebraic equations, from which a closed-form series solution can be obtained easily. The $k^{\text {th }}$ differential transformation of an analytical function $u(y)$ is defined as (For details See Zhou [14])

$$
U(k)=\frac{1}{k !}\left[\frac{d^{k} u(y)}{d y^{k}}\right]_{y=y_{0}},
$$

and the inverse differential transformation is given by

$$
u(y)=\sum_{k=0}^{\infty} U(k)\left(y-y_{0}\right)^{k}
$$

Combining Eqs (3.11) and (3.12), we obtain

$$
u(y)=\left.\sum_{k=0}^{\infty} \frac{\left(y-y_{0}\right)^{k}}{k !} \frac{d^{k} u(y)}{d y^{k}}\right|_{y=y_{0}} .
$$

From Eqs (3.10)-(3.12), it can be seen that the differential transformation method is derived from Taylor's series expansion. In real applications, $u(y)=\sum_{k=N}^{\infty} U(k)\left(y-y_{0}\right)^{k}$ is very small and can be neglected when $N$ is sufficiently large. So $u(y)$ can be expressed by a finite series, and Eq.(3.11) may be written as 


$$
u(y)=\sum_{k=0}^{N} U(k)\left(y-y_{0}\right)^{k}
$$

where the value of $N$ depends on the convergence requirement in real applications. Table 1 lists the basic mathematical operations frequently used in the following analysis.

\subsubsection{Formulation of the DTM}

From Tab.1, the transformed form of Eq.(2.13) with the corresponding initial conditions are

$$
\begin{aligned}
& U(k+4)=\frac{1}{(k+1)(k+2)(k+3)(k+4)}\left(E^{2}(k+1)(k+2) U(k+2)+\right. \\
& \left.+\lambda B r \sum(k-r)(k-r+1) U(k-r) U(k-r+1)+48 E^{2} \delta(k)\right)
\end{aligned}
$$

$U(0)=a_{1}, U(1)=a_{2}, U(2)=a_{3}, U(3)=a_{4}$, where $a_{1}, a_{2}, a_{3}$ and $a_{4}$ are constants and are evaluated using condition (2.14).

Table 1. The operations for the one-dimensional differential transform method.

\begin{tabular}{|c|c|}
\hline Original function & Transformed function \\
\hline$y(x)=g(x) \pm h(x)$ & $Y(k)=G(k) \pm H(k)$ \\
\hline$y(x)=\alpha g(x)$ & $Y(k)=\alpha G(k)$ \\
\hline$y(x)=\frac{d g(x)}{d x}$ & $Y(k)=(k+l) G(k+1)$ \\
\hline$y(x)=\frac{d^{2} g(x)}{d x^{2}}$ & $Y(k)=(k+l)(k+2) G(k+2)$ \\
\hline$y(x)=g(x) h(x)$ & $Y(k)=\sum_{l=0}^{k} G(l) H(k-l)$ \\
\hline$y(x)=x^{m}$ & $Y(k)=\delta(k-m)=\left\{\begin{array}{l}1, \text { if } k=m \\
0, \text { if } k \neq m\end{array}\right.$ \\
\hline
\end{tabular}

\subsection{Perturbation method}

Equation (2.13) along with boundary conditions (2.14) is nonlinear and hence it is difficult to find the closed form solutions. We employ a perturbation series method by using the dimensionless parameter

$$
\varepsilon=\operatorname{Br} \lambda=\operatorname{Re} \operatorname{Pr} \frac{\beta g D}{C_{p}}
$$

as the perturbation parameter. The solution of Eq.(2.13) can be expressed by the perturbation series method and is given by 


$$
u(y)=u_{0}(y)+\varepsilon u_{1}(y)+\varepsilon^{2} u_{2}(y)+\cdots \cdots \cdots=\sum_{n=0}^{\infty} \varepsilon^{n} u_{n}(y) .
$$

The second and higher order terms of $\varepsilon$ gives a correction to $u_{0}, u_{1}$ accounting for the viscous dissipation effects.

Isothermal-isothermal walls $\left(T_{1}-T_{2}\right)$

Using Eq.(3.16) in Eqs (2.13) and (2.14) and equating the coefficients of like powers of $\varepsilon$, we obtain the boundary value problems for $n=0$ and $n=1$ as

$$
\begin{aligned}
& \frac{d^{4} u_{0}}{d y^{4}}=E^{2} \frac{d^{2} u_{0}}{d y^{2}}+48 E^{2}, \\
& \frac{d^{4} u_{1}}{d y^{4}}=E^{2} \frac{d^{2} u_{1}}{d y^{2}}+\left(\frac{d u_{0}}{d y}\right)^{2}, \\
& u_{0}\left(-\frac{1}{4}\right)=u_{0}\left(\frac{1}{4}\right)=0,\left.\quad \frac{d^{2} u_{0}}{d y^{2}}\right|_{y=-\frac{1}{4}}=-48+\frac{\lambda R_{T}}{2},\left.\quad \frac{d^{2} u_{0}}{d y^{2}}\right|_{y=\frac{1}{4}}=-48-\frac{\lambda R_{T}}{2}, \\
& u_{1}\left(-\frac{1}{4}\right)=u_{1}\left(\frac{1}{4}\right)=0,\left.\quad \frac{d^{2} u_{1}}{d y^{2}}\right|_{y=-\frac{1}{4}}=0,\left.\quad \frac{d^{2} u_{1}}{d y^{2}}\right|_{y=\frac{1}{4}}=0 .
\end{aligned}
$$

Equation (3.17) is an ordinary linear differential equation and its exact solution can be found. This solution obviously coincides with the solution of Eq.(2.13) in case of $\mathrm{Br}=0$. The solution of Eq.(3.17) subjected to the boundary conditions (3.19) is

$$
u_{0}=\frac{3}{2}-24 y^{2}+\frac{2 R_{T} \lambda}{E^{2}}\left(y-\frac{\sinh (E y)}{4 \sinh (E / 4)}\right) .
$$

Equation (3.18) can also be solved exactly by using Eq.(3.17) and boundary conditions given by Eq.(3.20) and the solution is

$$
\begin{aligned}
& u_{1}=C_{1}+C_{2} y+C_{3} \cosh (E y)+C_{4} \sinh (E y)+l_{4} y^{2}+l_{5} y^{3}+l_{6} y^{4}+ \\
& +l_{7} y \cosh (E y)+l_{8} y \sinh (E y)+l_{9} y^{2} \cosh (E y)+l_{10} \cosh (2 E y) .
\end{aligned}
$$

An evaluation of the solution for $n \geq 2$ becomes complicated and hence we will neglected all the terms for $n \geq 2$ in Eq.(3.16). Therefore the solution becomes

$$
u=u_{0}+\varepsilon u_{1} .
$$

The dimensionless temperature field is obtained from Eq.(2.15) by substituting the velocity fields from Eqs (3.21) and (3.22) 


$$
\begin{aligned}
& \theta=\left[\frac{R_{T}}{2} \operatorname{cosech}(E / 4) \sinh (E y)+\right. \\
& \left.-\frac{\varepsilon}{\lambda}\left(\begin{array}{l}
2 l_{4}+6 l_{5} y+12 l_{6} y^{2}+\left(C_{3} E^{2}+l_{7} E^{2} y+2 l_{8} E+2 l_{9}+l_{9} E^{2} y^{2}\right) \cosh (E y)+ \\
+\left(C_{4} E^{2}+l_{8} E^{2} y+2 l_{7} E+2 l_{9}+4 l_{9} E y\right) \sinh (E y)+4 E^{2} l_{10} \cosh (2 E y)
\end{array}\right)\right] .
\end{aligned}
$$

\section{Isoflux-isothermal $\left(q_{1}-T_{2}\right)$ walls}

For this case, the thermal boundary conditions for the channel walls can be written in the dimensional form as

$$
\begin{gathered}
q_{1}=-\left.k \frac{d T}{d Y}\right|_{Y=-\frac{L}{2}}, \\
T\left(\frac{L}{2}\right)=T_{2} .
\end{gathered}
$$

The dimensionless form of the above equations can be obtained by using Eq.(2.8) with $\Delta T=q_{1} D / k$ to give

$$
\begin{gathered}
\left.\frac{d \theta}{d y}\right|_{y=-\frac{1}{4}}=-1, \\
\theta\left(\frac{1}{4}\right)=R_{q t}
\end{gathered}
$$

where $R_{q t}=\left(T_{2}-T_{0}\right) / \Delta T$ is the thermal ratio parameter for the isoflux-isothermal case.

Differentiating Eq.(2.2) with respect to $Y$ with $d P / d X=A$ gives

$$
\frac{d^{3} U}{d Y^{3}}+\frac{\beta g}{v} \frac{d T}{d Y}=0
$$

Equation (3.29) is non-dimensionalized by using Eq.(2.8) to give

$$
\frac{d^{3} u}{d y^{3}}+\lambda \frac{d \theta}{d y}=0
$$

Evaluating Eq.(3.30) at the left wall $(y=-1 / 4)$ yields

$$
\frac{d^{3} u}{d y^{3}}\left(-\frac{1}{4}\right)=\lambda
$$

The other boundary condition at the right wall can be shown to be the same as that given for the isothermal-isothermal wall with $R_{T}$ replaced by $R_{q t}$ such that 


$$
\frac{d^{2} u}{d y^{2}}\left(\frac{1}{4}\right)=-48-\frac{\lambda R_{q t}}{2}
$$

\section{Isothermal-isoflux $\left(T_{1}-q_{2}\right)$ walls}

For this case, the thermal boundary conditions for the channel walls can be written in the dimensional form as

$$
\begin{aligned}
& q_{2}=-\left.k \frac{d T}{d Y}\right|_{Y=\frac{L}{2}}, \\
& T\left(-\frac{L}{2}\right)=T_{1} .
\end{aligned}
$$

The dimensionless form of the above equations can be obtained by using the Eq.(2.8) with $\Delta T=q_{2} D / k$ to give

$$
\begin{aligned}
& \left.\frac{d \theta}{d y}\right|_{y=\frac{1}{4}}=-1, \\
& \theta\left(-\frac{1}{4}\right)=R_{t q}
\end{aligned}
$$

where $R_{t q}=\left(T_{1}-T_{0}\right) / \Delta T$ is the thermal ratio parameter for the isothermal-isoflux case.

Similar to the procedure done in the previous section on isoflux-isothermal walls, the dimensionless form of the boundary conditions obtained from Eq.(2.2) and by using Eq.(3.35) is

$$
\frac{d^{3} u}{d y^{3}}\left(\frac{1}{4}\right)=\lambda
$$

The other boundary condition at the right wall can be shown to be the same as that given for the isothermal-isothermal wall with $R_{T}$ replaced by $R_{t q}$ such that

$$
\frac{d^{2} u}{d y^{2}}\left(-\frac{1}{4}\right)=-48+\frac{\lambda R_{t q}}{2}
$$

For all practical purposes, the important physical quantities of interest are the skin friction (at the right wall and left wall) and Nusselt number at the plates defined as

$$
\tau_{1}=\left.\frac{d u}{d y}\right|_{y=-1 / 4} \quad \text { and } \quad \tau_{2}=\left.\frac{d u}{d y}\right|_{y=1 / 4},
$$




$$
\begin{aligned}
& \mathrm{Nu}_{1}=\left.\frac{D}{R_{T}(T(L / 2)-T(-L / 2))+\left(1-R_{T}\right) \Delta T} \frac{d T}{d Y}\right|_{Y=-L / 2}, \\
& \mathrm{Nu}_{2}=\left.\frac{D}{R_{T}(T(L / 2)-T(-L / 2))+\left(1-R_{T}\right) \Delta T} \frac{d T}{d Y}\right|_{Y=L / 2} .
\end{aligned}
$$

By employing Eq.(2.8) on Eqs (3.40) and (3.41), we write

$$
\begin{aligned}
& \mathrm{Nu}_{1}=\left.\frac{1}{R_{T}(\theta(1 / 4)-\theta(-1 / 4))+\left(1-R_{T}\right)} \frac{d \theta}{d y}\right|_{y=-1 / 4}, \\
& \mathrm{Nu}_{2}=\left.\frac{1}{R_{T}(\theta(1 / 4)-\theta(-1 / 4))+\left(1-R_{T}\right)} \frac{d \theta}{d y}\right|_{y=1 / 4} .
\end{aligned}
$$

The dimensionless form of mean velocity is defined as

$$
\bar{u}=\int_{-1 / 4}^{1 / 4} u(y) d y
$$

The constants which appeared in the solutions are not presented, due to brevity.

\section{Results and discussion}

The nonlinear ordinary differential Eq.(2.13) with boundary conditions (2.14) is solved analytically using the Differential Transform Method (DTM) and regular perturbation method (PM). The analytical solutions are found using the regular perturbation method with the product of the mixed convection parameter $\lambda(=\mathrm{Gr} / \mathrm{Re})$ and Brinkman number $\mathrm{Br}$ as the perturbation parameter. The perturbation solutions are valid only for small values of the perturbation parameter $\varepsilon(<1)$. As there are no exact solutions for nonlinear equations, a comparison of analytical solutions is conducted and also the results are compared with the available data.

In order to understand the flow characteristics, we present a comprehensive study of our findings in tabular and graphical forms together with the discussion and their interpretations. As our objective is to develop a better understanding of the effects of radiation on the flow field, we choose to present the shear stress and Nusselt number at the plates and the velocity, temperature field between the two vertical plates for a range of the mixed convective parameter $\lambda$, the radiation parameter $E$, the perturbation parameter $\varepsilon$, and the Brinkman number Br. The parameters $\lambda, \mathrm{Br}, E$ and $\varepsilon$ are fixed as $\pm 500,0.1,5.0$, and \pm 0.1 respectively except the varying one. The flow field in the case of asymmetric heating $\left(R_{T}=1\right)$ and symmetric heating $\left(R_{T}=0\right)$ for different special cases with isothermal-isothermal, isoflux-isothermal, and isothermal-isoflux are obtained and presented in Figs 2 to 11.

Plots of $u$ versus $y$ for asymmetric heating $\left(R_{T}=1\right)$ conditions for $\lambda= \pm 500$ (both assisting and opposing flows) and $\varepsilon=0, \pm 8, \pm 12$ are shown in Fig.2 where it can be observed that the velocity field increases for the increasing values of $\varepsilon$ for the assisting flow and reverse effect is observed on the opposing flow. For large values of $\lambda$, flow reversal occurs at both the cold and hot walls. The effect of $\lambda$ and $\varepsilon$ on temperature is demonstrated in Fig.3. As $\varepsilon$ increases, the temperature increases for the 
assisting and opposing flow also, as we expected in the mixed convection when the value of $\varepsilon=0$ the profiles are linear due to the presence of radiation in the fluid, the nature of the temperature profiles is not linear. The effects of the radiation parameter $E$ on the velocity and temperature fields are presented in Figs 4 and 5 respectively. As the radiation parameter increases the velocity increases in the left half of the region and decreases in the right half of the region for the assisting flow whereas the reverse effect is observed on the opposing flow (See Fig.4). That is, for the opposing flow velocity decreases in the left half of the region and increases in the right half of the region as the radiation parameter increases. The variation of the radiation parameter $E$ on the temperature field is observed in Fig.5. The radiation parameter increases the temperature in the left half of the region and decreases in right half of the region. Figures 6 and 7 depict the variation of the radiation parameter on the velocity and temperature fields respectively for the isothermal-isothermal symmetric wall boundary conditions. It is observed from Fig. 6 that the effect of the radiation parameter on the velocity field is invariant for both assisting and opposing flows. The effect of the radiation parameter is to decreases the temperature field and it is observed that the effect is similar for both assisting and opposing flows. Figures 8 to 11 elucidates the effect of the radiation parameter for the assisting and opposing flow for isofluxisothermal and isothermal-isoflux for asymmetric heating. The velocity increases for the opposing flow and decreases for the assisting flow as the radiation parameter increases for the cases of isofluxisothermal wall condition (See Fig.8). As the radiation parameter increases, the temperature decreases for both assisting and opposing flows as seen in Fig.9. It is also observed that the effect is highly significant near the cold wall for isoflux-isothermal wall condition. The effects of the radiation parameter on velocity and temperature for isothermal-isoflux wall condition are depicted in Figs 10 and 11 respectively. The effect of the radiation parameter velocity is opposite to that of isoflux-isothermal wall conditions. The temperature increases for both assisting and opposing flows but the magnitude of promotion is significant for the opposing flow as seen in Fig.11. It is also observed that the effect is highly significant near the hot wall.

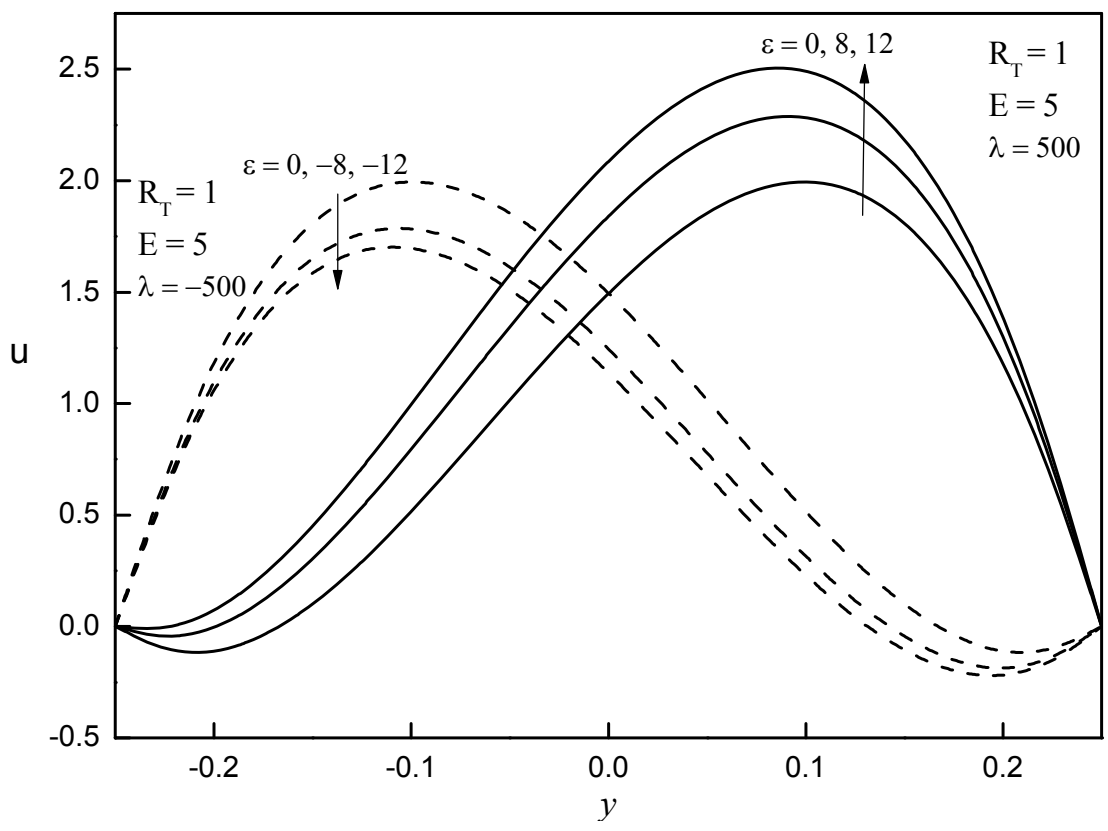

Fig.2. Velocity profiles for different values of $\lambda$ and $\varepsilon$. 


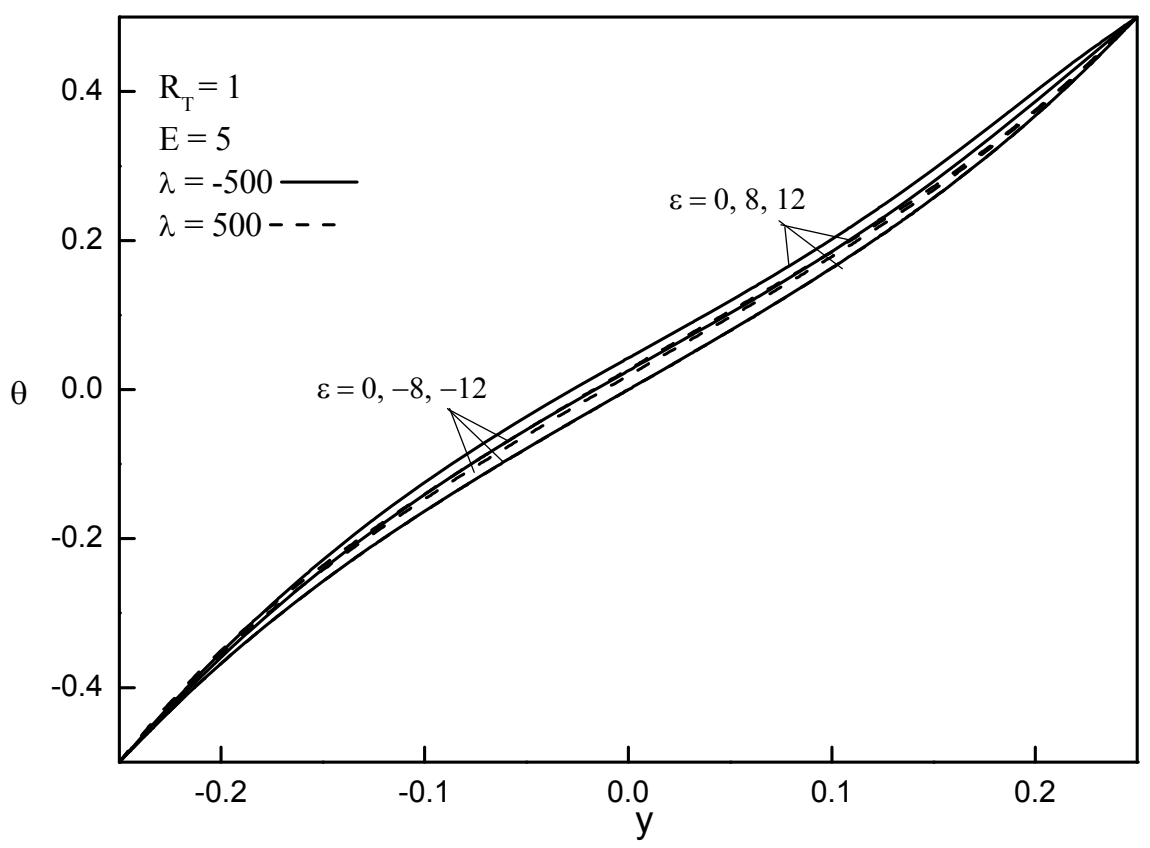

Fig.3. Temperature profiles for different values of $\lambda$ and $\varepsilon$.

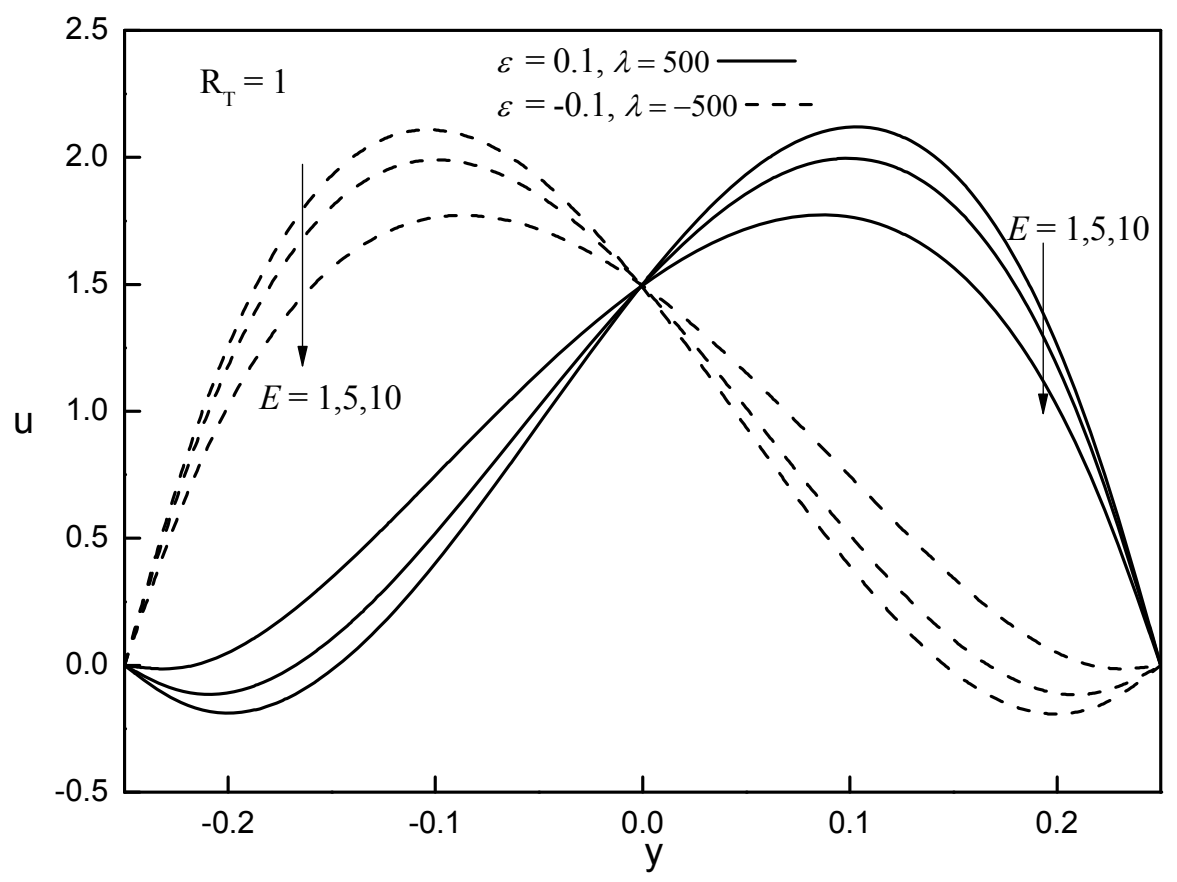

Fig.4. Velocity profiles for different values of the radiation parameter $E$. 


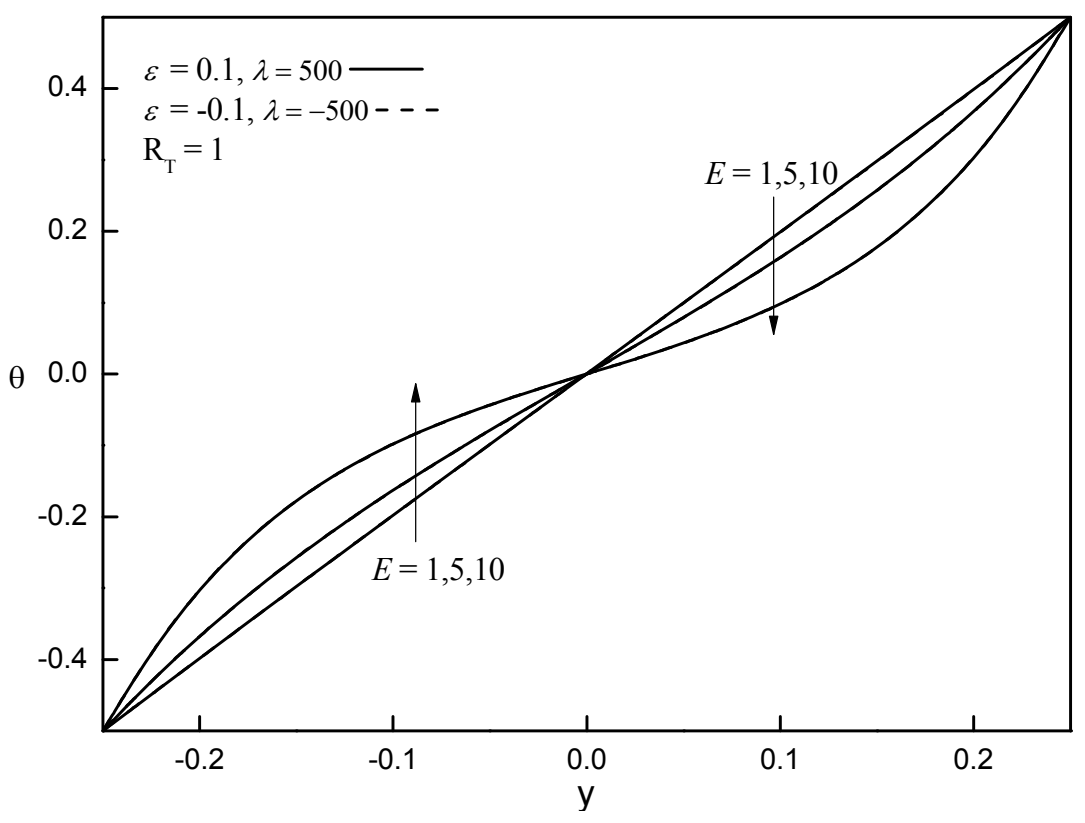

Fig.5. Temperature profiles for different values of the radiation parameter $E$.

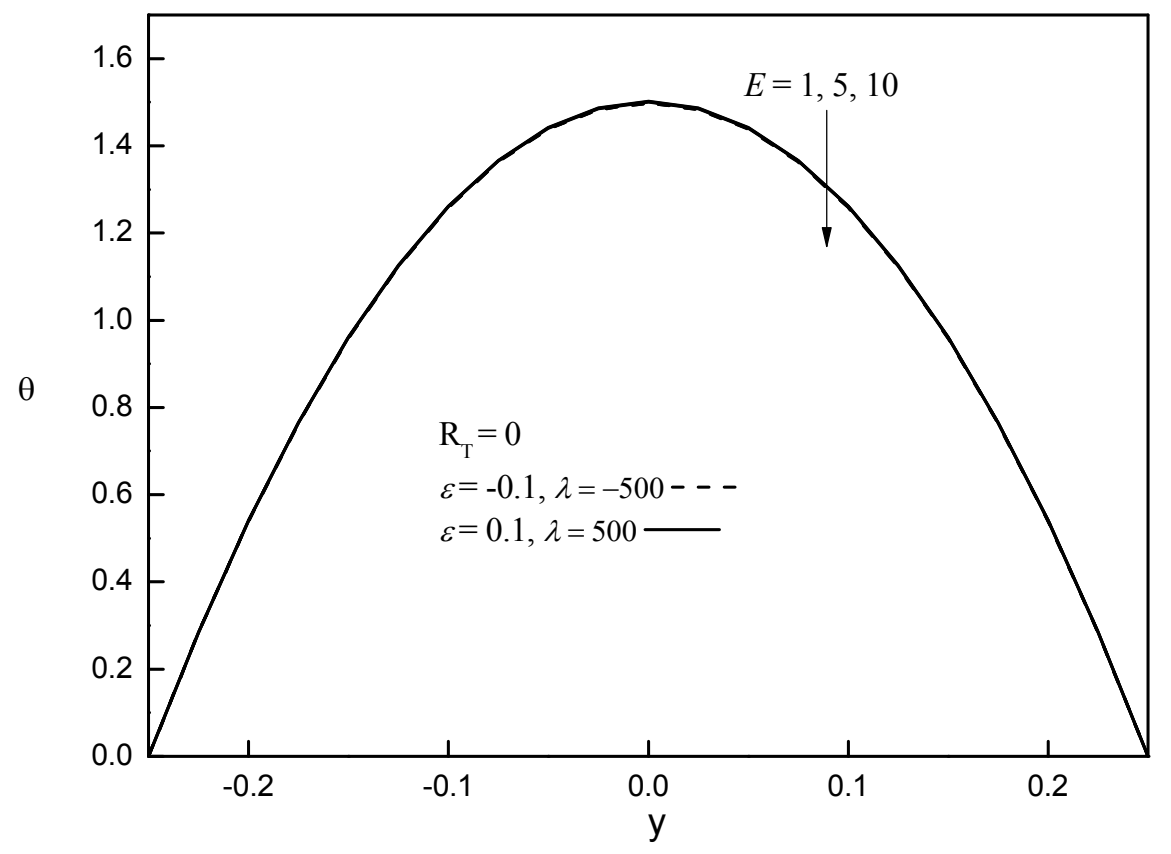

Fig.6. Velocity profiles for different values of the radiation parameter $E$ for $R T=0$. 


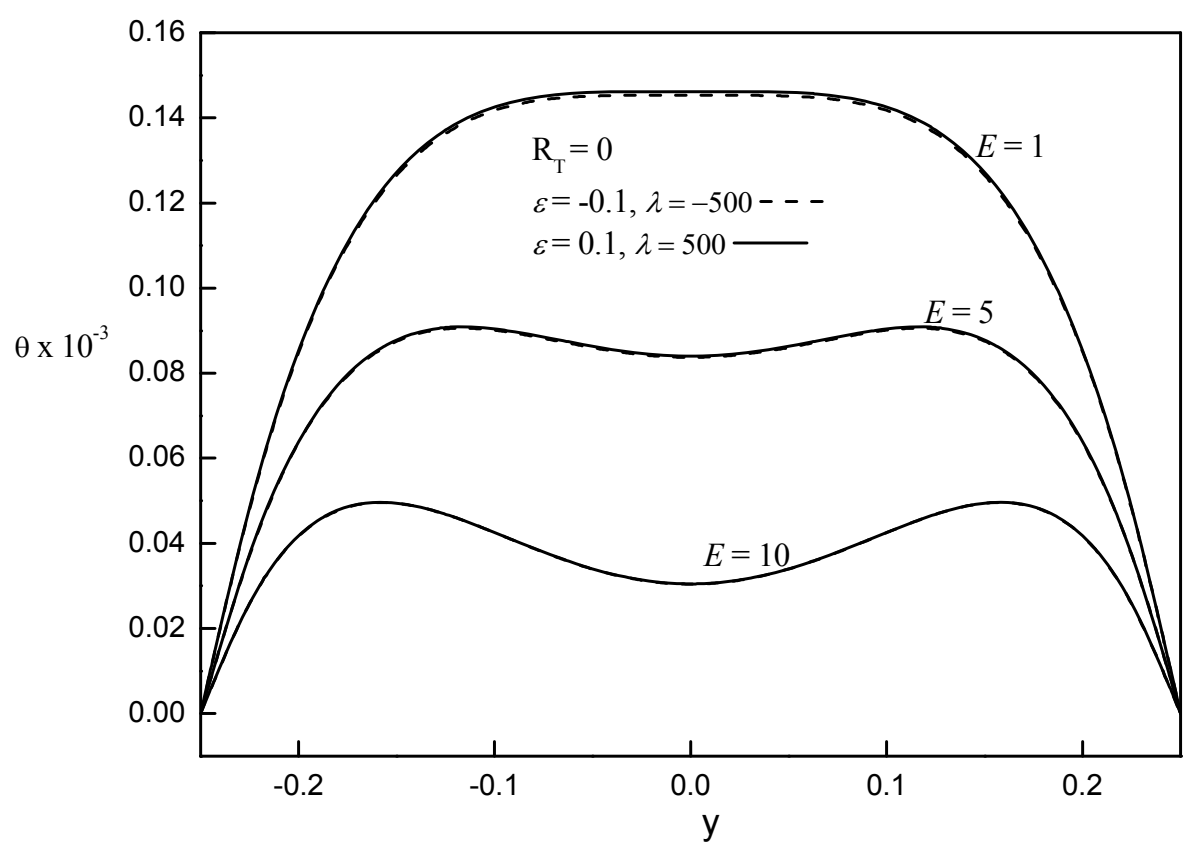

Fig.7. Temperature profiles for different values of the radiation parameter $E$ for $R_{T}=0$.

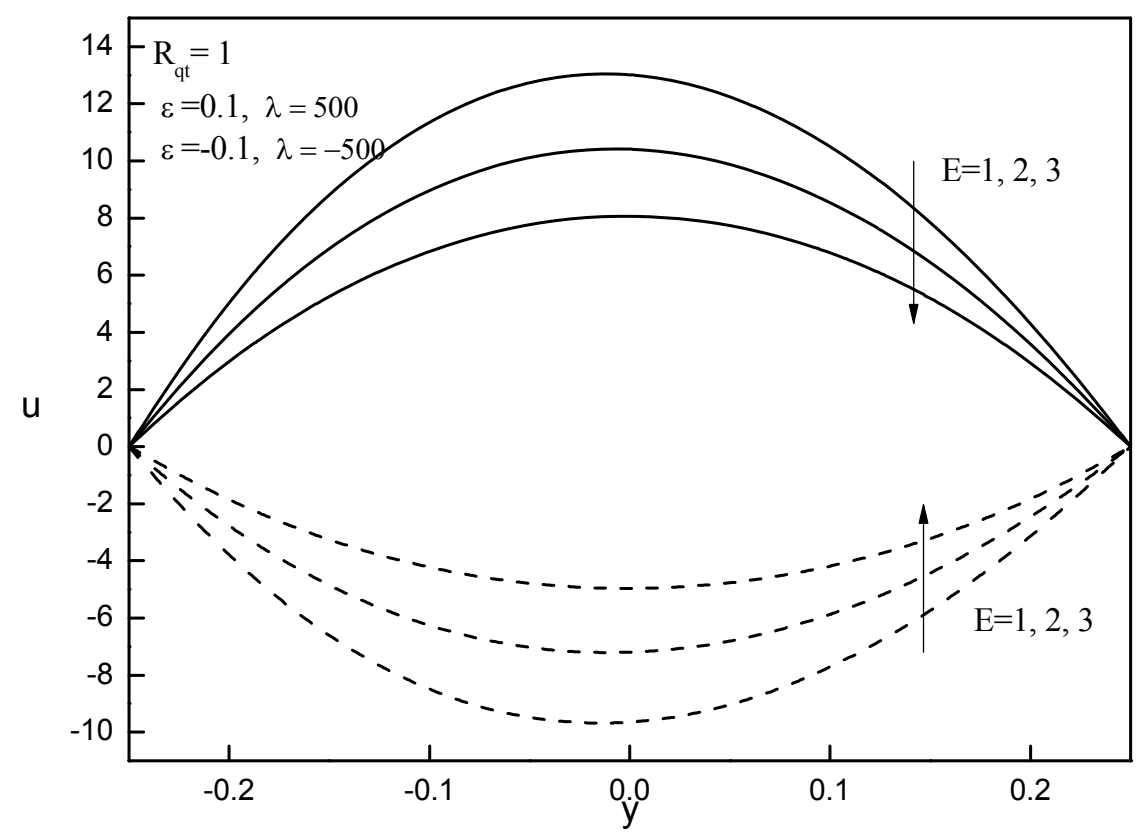

Fig.8. Velocity profiles for different values of the radiation parameter $E$ of isoflux-isothermal. 


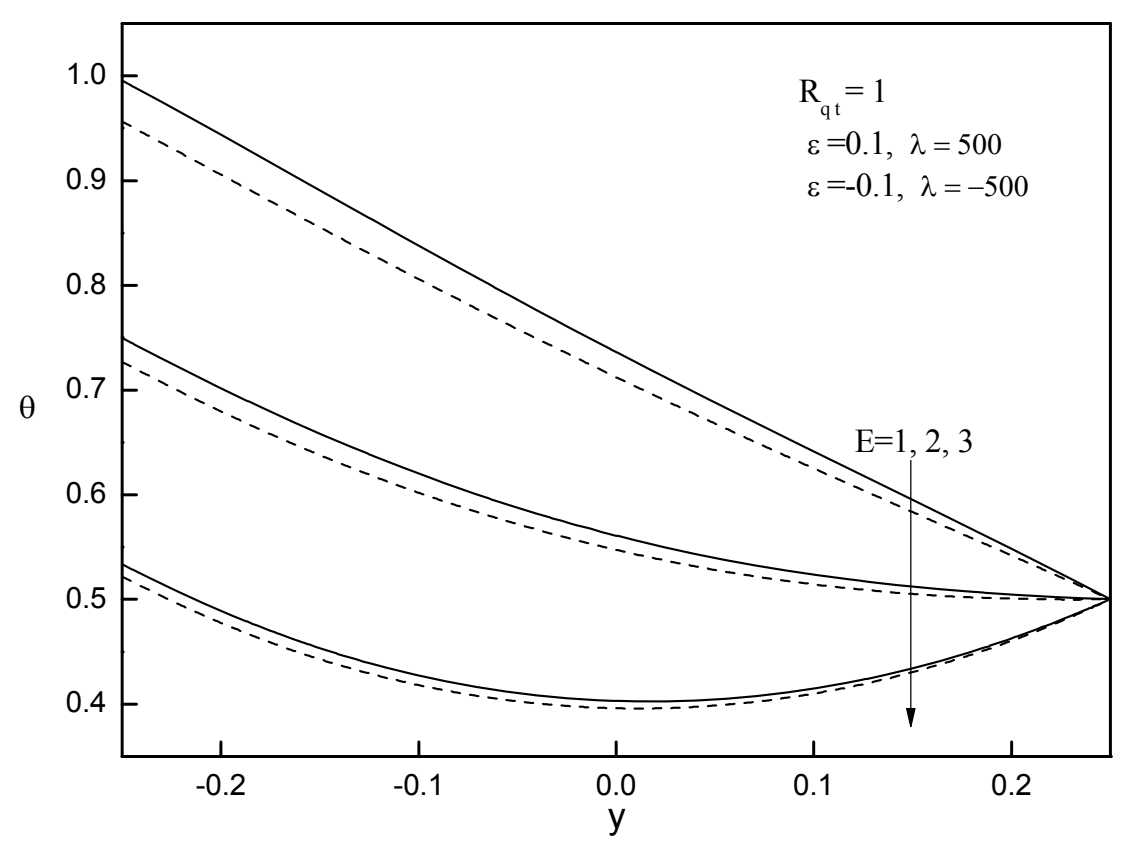

Fig.9. Temperature profiles for different values of the radiation parameter $E$ of isoflux-isothermal.

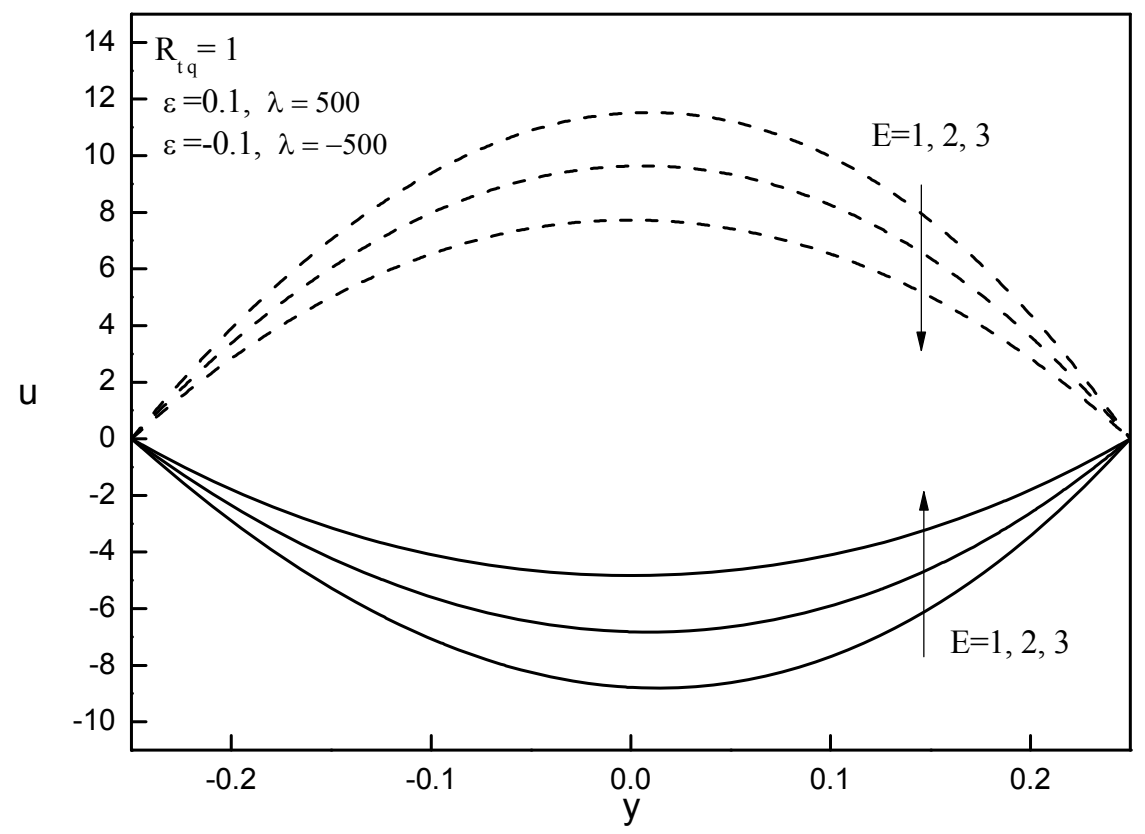

Fig.10. Velocity profiles for different values of the radiation parameter $E$ of isothermal-isoflux. 


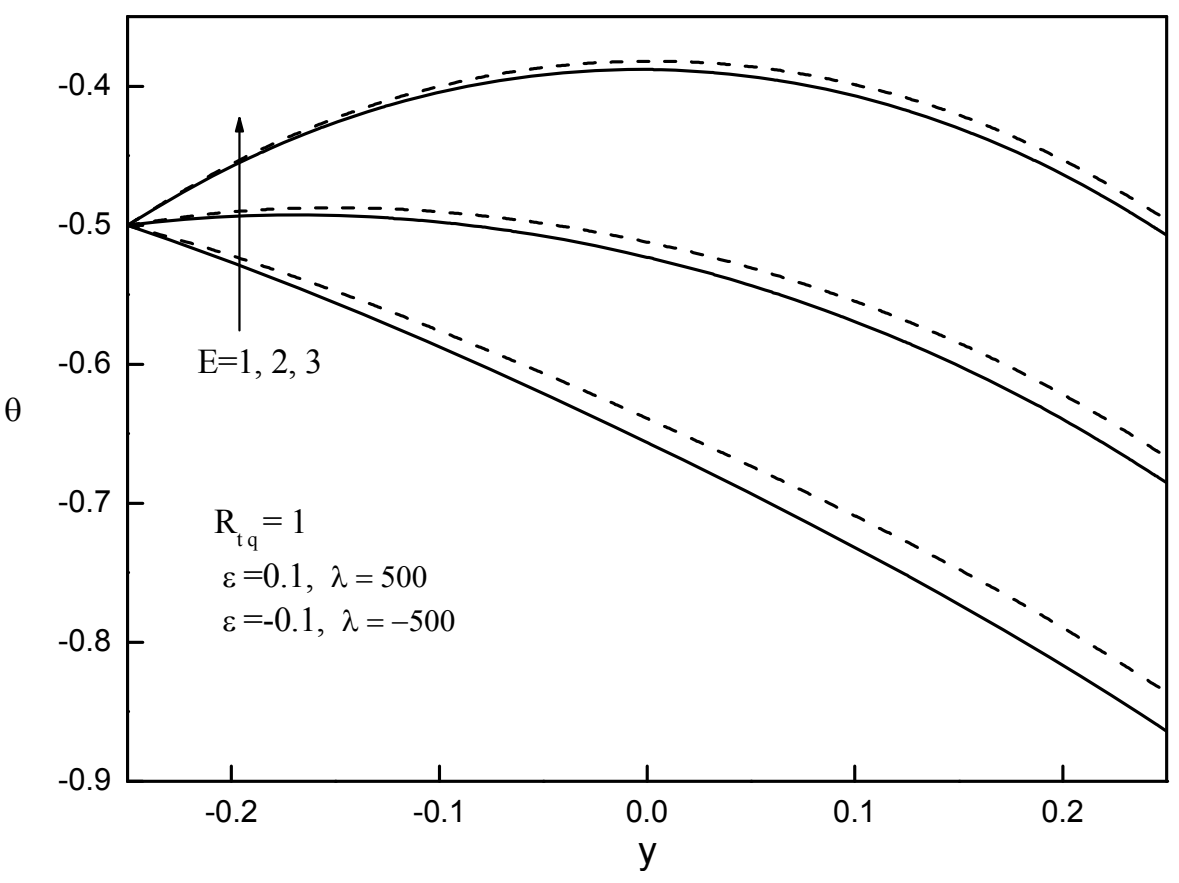

Fig.11. Temperature profiles for different values of the radiation parameter $E$ of isothermal-isoflux.

Table 2 shows the velocity and temperature distribution with DTM and the analytically solution in the presence and absence $(\varepsilon \neq 0$ and $\varepsilon=0)$ of viscous dissipation with $E=2, R_{T}=1$ and $\lambda=500$. It is observed that this method provides more accurate results. Moreover, it is clear from the table that as $\varepsilon$ increases, the error with the perturbation method increases. The Nusselt number obtained in the absence of the radiation effect is in good agreement with Barletta [3] for both the assisting and opposing flow. (See Tab.3). Results for the mass flow rate and shear stress are presented in Tab.4a for various values of $\lambda, \varepsilon$. By a careful scrutiny into Tab.4, one can observe that as $\varepsilon$ increases the skin friction increases in magnitude for both the assisting and opposing flow. In other words, the Boussinesq approximation gives small but acceptable errors for small temperature differences (i.e., small values of $\varepsilon)$, but it gives substantial errors for high temperature differences (i.e., large values of $\varepsilon$ ). The mass flow also increases as $\varepsilon$ and $\lambda$ increase. Table 5 shows the effect of the Nusselt number at the left wall $\mathrm{Nu}_{-}$and at the right wall $\mathrm{Nu}_{+}$. It is clear that as the perturbation parameter $\varepsilon$ increases, the Nusselt number decreases at the left wall and increases at the right wall for both the positive and negative values of $\lambda$. Also it is observed that the Nusselt number increases for increasing values of $\lambda$ (positive values) at the right wall and decreases at the left wall whereas a reverse effect is observed for negative values of $\lambda$. 

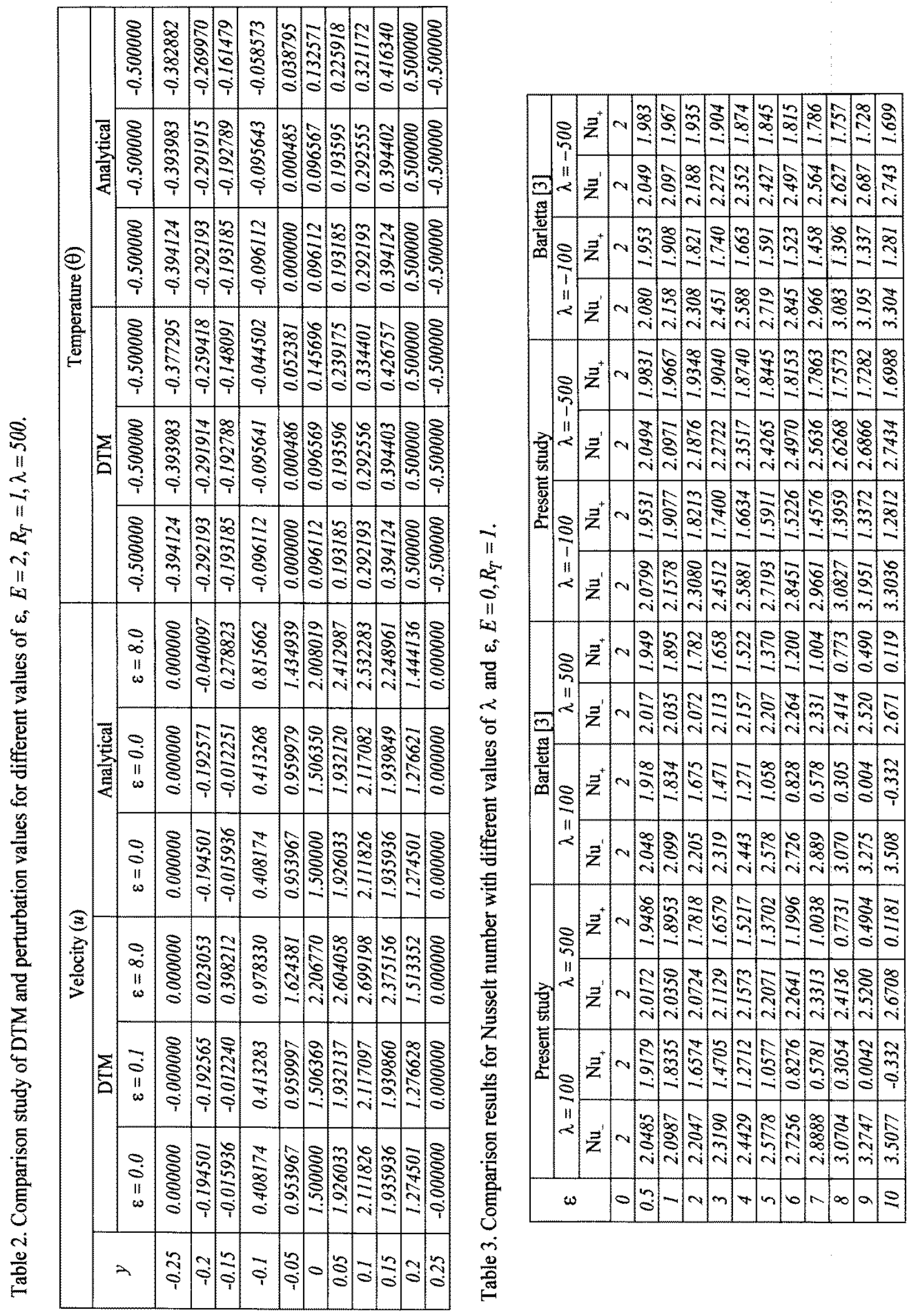

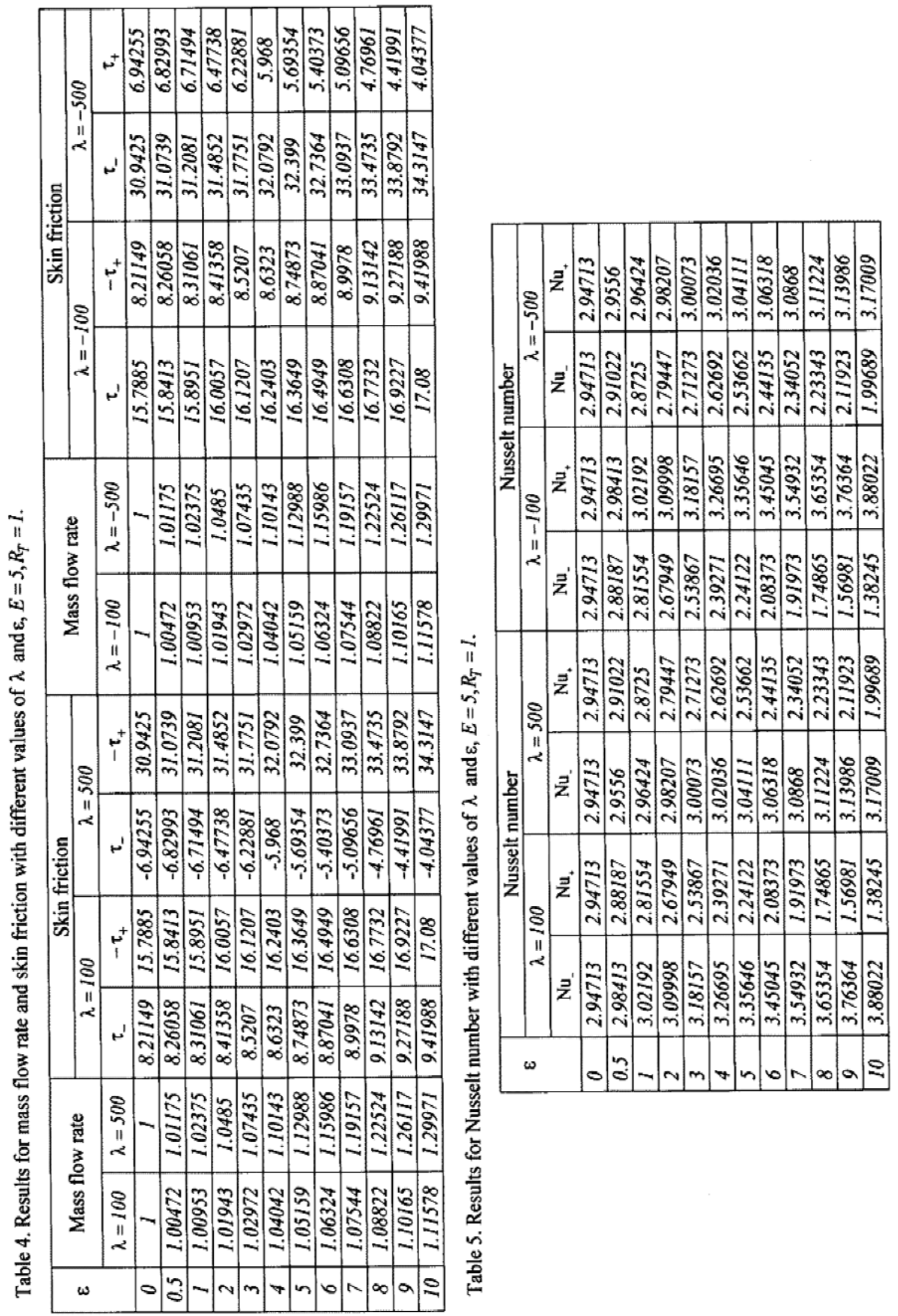


\section{Conclusion}

In the present study, the DTM and perturbation method were used to find analytical solutions of a mixed convection problem in the presence of radiation effect and viscous dissipation. It was found that the DTM is a powerful method for solving problems consisting of systems of nonlinear differential equations. In this paper, a reliable algorithm is presented based on the DTM to solve highly nonlinear equations. A comparison was made between perturbation method solutions, available published results and the present approximate solutions. The numerical results indicate that the DTM is more accurate in comparison to the shown methods. The method has been applied directly without requiring linearization, discretization, or perturbation. The obtained results certify the reliability of the algorithm and give it a wider applicability to nonlinear differential equations. Also, some of the major observations are: the velocity and temperature increase for mixed convection parameter and perturbation parameter whereas it decreases for increasing the radiation parameter.

\section{Acknowledgments}

The author would like to thank UGC-New Delhi for the financial support under UGC-Minor Research Project (F. No. 42-995/2013 (SR)).

\section{Nomenclature}

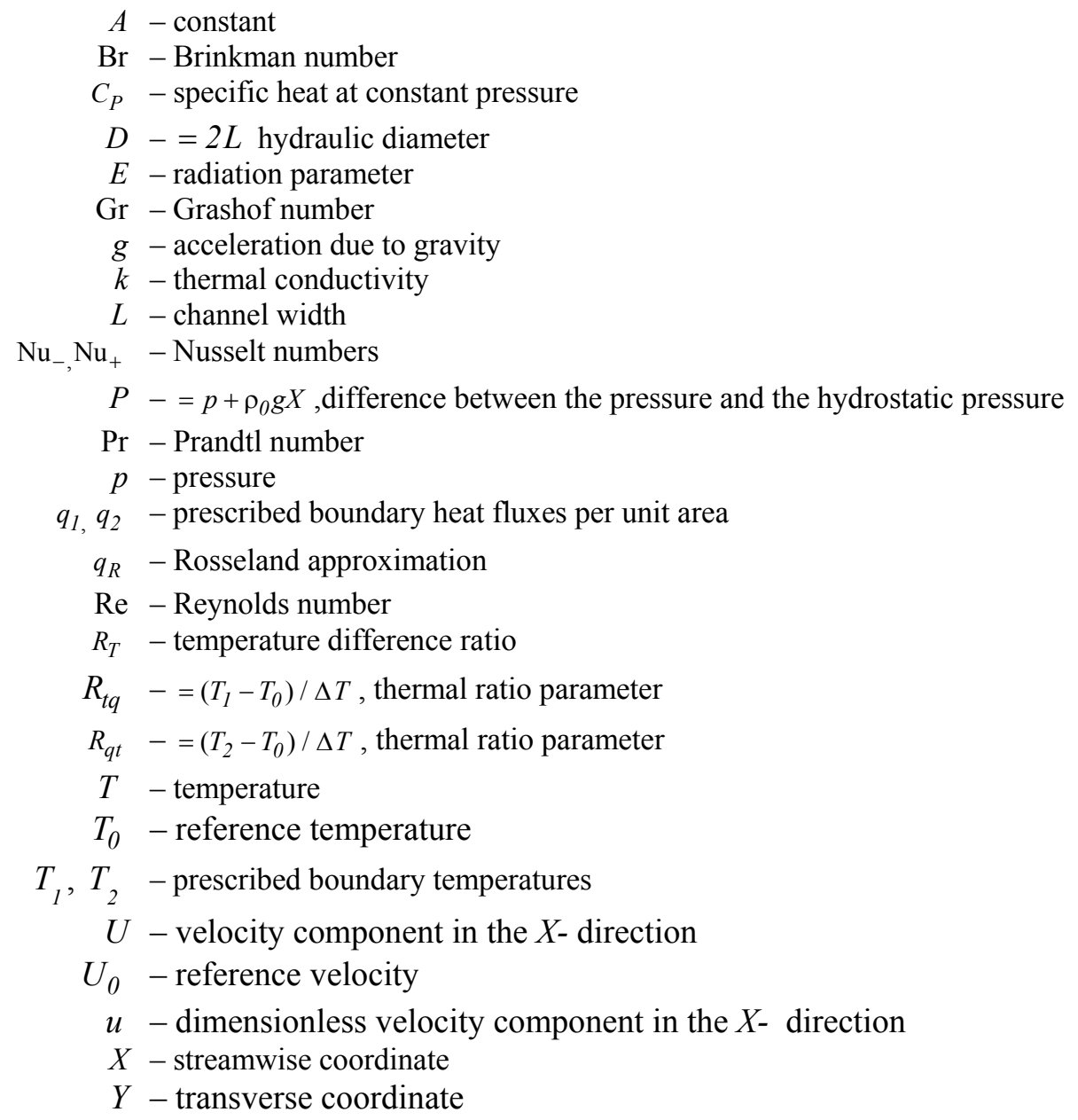


$y$ - dimensionless transverse coordinate

$\alpha-$ thermal diffusivity

$\beta$-thermal expansion coefficient

$\Delta T$ - reference temperature difference

$\varepsilon$-dimensionless parameter

$\theta$-dimensionless temperature

$\lambda$ - dimensionless parameter

$\mu-$ dynamic viscosity

$v$ - kinematic viscosity

$\rho$ - mass density

$\rho_{0} \quad$ - value of the mass density when $T=T_{0}$

$\sigma$ - Stefan-Boltzman constant

$\chi$ - mean absorption constant

\section{References}

[1] Aung W. and Worku G. (1986): Theory of fully developed combined convection including flow reversal. - ASME J. of Heat and Transfer, vol.108, pp.485-488.

[2] Zanchini E. (1998): Effect of viscous dissipation on mixed convection in a vertical channel with boundary conditions of the third kind. - Int. J. of Heat and Mass Transfer, vol.41, pp.3949-3959.

[3] Barletta A. (1998): Laminar mixed convection with viscous dissipation in a vertical channel. - Int. J. Heat and Mass Transfer, vol.41, pp.3501-3513.

[4] Boulama K. and Galanis N. (2004): Analytical solution for fully developed mixed convection between parallel vertical plates with heat and mass transfer. - J. of Heat Transfer, vol.126, pp.381-388.

[5] Barletta A., Magyari E. and Keller B. (2005): Dual mixed convection flows in a vertical channel. - Int. J. of Heat and Mass Transfer, vol.48, pp.4835-4845.

[6] Prasad K.V., Vaidya H. and Vajravelu K. (2015): MHD mixed convection heat transfer in a vertical channel with temperature-dependent transport properties. - Journal of Applied Fluid Mechanics, vol.8, No.4, pp.693-701.

[7] Raptis A. (2001): Radiation and flow through a porous medium. - J. of Porous Media, vol.4, pp.271-273.

[8] Bakier A.Y. (2001): Thermal radiation effects on mixed convection from vertical surfaces in saturated porous media. - Int. Comm. of Heat and Mass Transfer, vol.28, pp.243-248.

[9] Raptis A. and Perdikis C. (2004): Unsteady flow through a highly porous medium in the presence of radiation.Transport Porous Media, vol.57, pp.171-179.

[10] Grosan T. and Pop I. (2007): Thermal radiation effect on fully developed mixed convection flow in a vertical channel. - Technische Mechanik, vol.27, pp.37-47.

[11] Bég O.A., Zeuco J., Takhar H.S. and Bég T.A. (2008): Network numerical simulation of impulsively - started transient radiation-convection heat and mass transfer in a saturated Darcy-Forchheimer porous medium. - Non Linear Analysis: Modeling and Control, vol.13, pp.281-303.

[12] Ghosh S.K. and Bég O.A. (2008): Theoretical analyses of radiative effects on transient free convection heat transfer past a hot vertical surface in porous media. - Non Linear Analysis: Modelling and Control, vol.13, pp.419-432.

[13] Özişik M.N. (1987): Interaction of radiation with convection. - In: Hand book of Single-Phase Convective Heat Transfer (Kakaç, S.; Shah, R.K.; Aung W., Eds.). Wiley, New York, pp.19.1-19.3.

[14] Zhou J.K. (1986): Differential transform and its applications for electrical circuits. - Wuhan, Huarjung University Press. 
[15] Arikoglu A. and Ozkol I. (2010): Vibration analysis of composite sandwich beams with viscoelastic core by using differential transform method. - Composite Structures, vol.92, pp.3031-3039.

[16] Bert C.W. (2002): Application of differential transforms method to heat conduction in tapered fins. - ASME Journal of Heat Transfer, vol.124, pp.208-209.

[17] Chu H.P. and Chen C.L. (2008): Hybrid differential transform and finite difference method to solve the nonlinear heat conduction problem. - Comm. in Nonlinear Science and Numerical Simulation, vol.13, pp.1605-1614.

[18] Chu H.P. and Lo C.Y. (2008): Application of the hybrid differential transform-finite difference method to nonlinear transient heat conduction problems. - Numerical Heat Transfer Part A, vol.53, pp.295-307.

[19] Joneidi A.A., Ganji D.D. and BabaelahiM. (2009): Differential transformation method to determine fin efficiency of convective straight fins with temperature dependent thermal conductivity. - Int. Comm. Heat and Mass Transfer, vol.36, pp.757-762.

[20] Rashidi M.M., Laraqi N. and Sadri S.M. (2010): A novel analytical solution of mixed convection about an inclined flat plate embedded in a porous medium using the DTM-Padé. - Int. J. of Thermal Sciences, vol.49, pp.2405-2412.

[21] Hessameddin Yaghoobi and Mohsen Torabi (2011): The application of differential transformation method to nonlinear equations arising in heat transfer. - Int. Comm. in Heat and Mass Transfer, vol.38, pp.815-820.

[22] Baytaş A.C., Liaqat A., Groşan T. and Pop I. (2001): Conjugate natural convection in a square porous cavity. Heat Mass Transfer, vol.37, pp.467-473.

[23] Aung W. and Worku G. (1986): Developing flow and flow reversal in a vertical channel with asymmetric wall temperature. - ASME Journal Heat Transfer, vol.108, pp.299-304.

[24] Batchlor G.K. (1954): Heat transfer by free convection across a closed cavity between vertical boundaries at different temperatures. - Quarterly of Applied Mathematics, vol.12, pp.209-233.

[25] Cheng K.C. and Wu R.S. (1976): Viscous dissipation effects on convective instability and heat transfer in plane Poiseulle flow heated from below. - Applied Scientific Research, vol.32, pp.327-346.

Received: April 6, 2016

Revised: October 14, 2016 